\title{
Developing a High-Performance Quantum Chemistry Program with a Dynamic Scripting Language
}

\author{
Tomomi Shimazaki \\ RIKEN Advanced Institute for \\ Computational Science \\ Kobe, Hyogo 650-0047, Japan \\ t-shimazaki@riken.jp
}

\author{
Masatomo Hashimoto \\ RIKEN Advanced Institute for \\ Computational Science \\ Kobe, Hyogo 650-0047, Japan \\ m.hashimoto@riken.jp
}

\author{
Toshiyuki Maeda \\ RIKEN Advanced Institute for \\ Computational Science \\ Kobe, Hyogo 650-0047, Japan \\ tosh@riken.jp
}

\begin{abstract}
We discuss the use of dynamic programming languages such as Python to develop first-principles quantum chemistry programs for high-performance computing environments. Dynamic scripting programming languages, in general, have distinct advantages in terms of developer productivity over compiled languages such as $\mathrm{C} / \mathrm{C}++$ and Fortran, because of their ease of use and extensive libraries. Such "static" languages have clear performance advantages due to their optimizing compilers. In particular, we report on our experience developing a quantum chemistry program that attempts to combine the best of both worlds by using language binding techniques to bridge different programming languages.
\end{abstract}

\section{Categories and Subject Descriptors}

J.2 [Physical Sciences and Engineering]: Chemistry; D.1 [Programming Techniques]

\section{General Terms}

Algorithms, Languages, Performance

\section{Keywords}

Quantum chemistry, First-principles calculations, Dynamic scripting languages, Python, Binding techniques, Highperformance computing, Productivity, HPC usability

\section{Introduction}

First-principles quantum chemistry calculations are used across a wide of specialties to investigate the chemical and physical properties of molecules and materials in both science and engineering. Software packages such as NWChem [1] and SMASH [2] provide ready-to-use implementations of quantum chemistry theories for molecular simulations. However, these packages are not always sufficient for investigating complex molecules and materials. For these purposes, one may need to integrate various techniques such as molecular mechanics, molecular dynamics, parameter search simulations, quantum Monte Carlo method, and the density-matrix renormalization group method, some of which are beyond the scope of quantum chemistry. It is a demanding task to implement and integrate a variety of simulation techniques in general.

Moreover, modern massively parallel high-performance computing (HPC) environments, such as supercomputers, parallelcluster workstations, and general-purpose computing on graphics processing units have become essential for practical large-scale

Permission to make digital or hard copies of all or part of this work for personal or classroom use is granted without fee provided that copies are not made or distributed for profit or commercial advantage and that copies bear this notice and the full citation on the first page. Copyrights for components of this work owned by others than ACM must be honored. Abstracting with credit is permitted. To copy otherwise, or republish, to post on servers or to redistribute to lists, requires prior specific permission and/or a fee. Request permissions from Permissions@acm.org. SE-HPCCSE2015, November 15-20, 2015, Austin, TX, USA

(C) 2015 ACM. ISBN 978-1-4503-4012-0/15/11 $\$ 15.00$

DOI: http://dx.doi.org/10.1145/2830168.2830170 simulations. Package developers therefore have to learn various computer architectures and parallel programming frameworks, such as OpenMP and the Message Passing Interface (MPI).

One way to improve this situation is by using appropriate programming languages to increase productivity [3, 4]. Dynamic scripting languages such as Python, Ruby, and Perl are preferred for the development of commodity programs such as web applications, mainly because of their ease of use and extensive libraries. In HPC environments, however, it is typically the case that only compiled languages such as $\mathrm{C} / \mathrm{C}++$ and Fortran are officially supported by vendors and operating facilities.

Although dynamic languages are not particularly popular in largescale scientific computing, a few projects have made some inroads. For example, NWChem provides Python application program interfaces, mostly written in Fortran [1]. PyQuante [5] is a quantum chemistry program written in Python that uses compiled languages for "rate determining" modules. LAMMPS [6] is a classical molecular dynamics package written in $\mathrm{C}++$ that provides Python modules. However, the main purpose of these interfaces is to make the respective programs callable from Python. They do not tell us how to integrate them (more specifically, integrate multiple existing programs) for practical simulations without sacrificing productivity or performance.

Here we discuss the use of dynamic programming languages to improve developer productivity with, and the usability of, quantum chemistry programs for HPC environments. We focus on Python [7] and detail a method to combine it and compiled languages based on language binding techniques to aid in the development of efficient quantum chemistry programs without performance degradation.

The paper is organized as follows: Section 2 briefly introduces a standard quantum chemistry algorithm. Section 3 explains our Python quantum chemistry program. Section 4 provides preliminary evaluations from our approach, and Section 5 concludes.

\section{Brief Introduction to the SCF Algorithm}

Although there are a number of computational approaches to quantum chemistry, in this paper we focus on the self-consistent field (SCF) method [8], which is one of the most popular quantum chemistry algorithms.

In Figure 1, we show the typical flow of an SCF simulation. First, molecular information (coordinates of the atoms in a target molecule, basis set, spin, and so on) is given as input data. The calculation conditions (simulation method, numerical thresholds, 


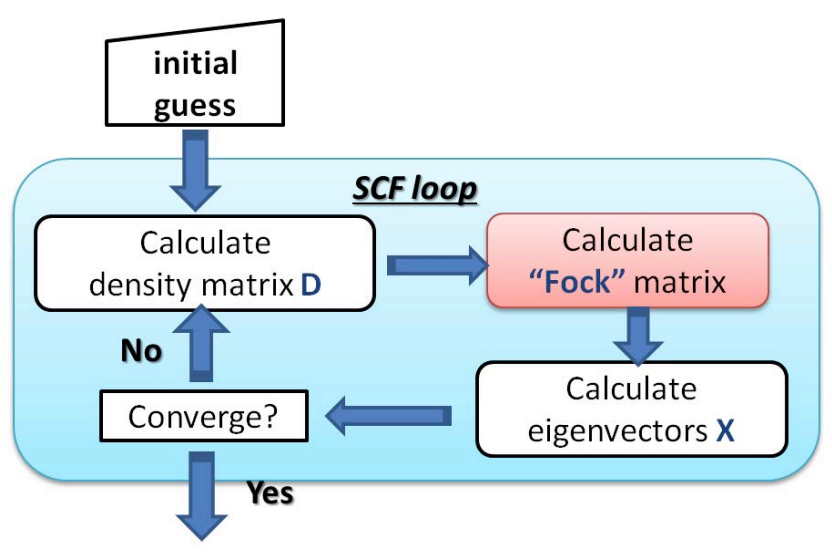

Figure 3. Flowchart for the SCF method.

In the scf function of Figure 2, the input elestr_guess is copied to ret_elestr. Next the core and overlap matrices, which are represented by $\mathrm{H}$ _core and $\mathrm{S}$ objects, are calculated by the cal_H_core and Cal_S functions from the moldata object. Then, scf reaches the loop statement, in which the density matrix $D$ is calculated by the cal_D function from the ret_elestr object. The matrix Fock is then calculated by the cal_Fock_sub function. The calculation of the Fock matrix is one of the most time-consuming parts of the SCF algorithm. In particular, calculation of the Hartree and exchange matrices [8] usually represent bottlenecks in quantum chemistry programs, because they require evaluation of electron-electron repulsion integrals. The cal_eigen function in the loop produces the eigenvectors and eigenvalues, which are used to update ret_elestr. The new ret_elestr object is employed to calculate a new density matrix $\mathrm{D}$ in the SCF loop, which is repeated until it converges, after which scf returns an ret_elestr object that contains the electronic structure of the target molecule.

\subsection{Productivity Aspects}

A variety of quantum chemistry theories are used to investigate different features of molecules and materials. Some of these can be implemented with by modifying the basic SCF loop illustrated above. In our experience, the dynamic programming approach has proved to be competitive with compiled languages, because the programs tend to be more concise and do not require the additional, repetitive, and time-consuming step of compilation. For example, we have studied the quantum nuclear effect on hydrogen atoms without the Born-Oppenheimer approximation [9], which requires an extra matrix. This can be implemented easily by inserting a function to add the matrix for the quantum nuclear effect in the SCF loop. Another case is the introduction of an approximate method based on auxiliary functions in order to reduce the computational burden of calculating the Hartree matrix, which is required for investigation of semiconductor materials [10]. This can readily be achieved by substituting an appropriate cal_Hartree for cal_Fock_sub in Figure 2.

\subsection{Performance Aspects}

Although there are benefits to the use of dynamic languages in the development of quantum chemistry programs, the results would be hopelessly inefficient if written entirely in this fashion. For example, several functions in Figure 2 (e.g., cal_S, cal_K, cal_NA, Cal_Hartee, and cal_Exchange) include many numerical integrations specialized for quantum chemistry and thus consume a great deal of CPU time. In addition, operations such as matrix addition and eigenvector calculations (e.g., mat add and cal_eigen) also demand significant numerical calculations. To make quantum chemistry programs practical and realistic in this context, these functions should be implemented in compiled languages such as $\mathrm{C} / \mathrm{C}++$ or Fortran. Thus, an integration of dynamic scripting languages with compiled languages is essential to the pursuit of the best of both worlds, that is, productivity and performance.

\section{Implementation Using Python}

In this section, we briefly explain how to implement the quantum chemistry algorithm described in the previous section using Python. First we present several binding techniques to integrate Python with $\mathrm{C} / \mathrm{C}++$ and Fortran, and then we introduce our implementation of the algorithm. In addition, we also provide some implementation notes relevant to our HPC environment.

\subsection{Binding Techniques}

Python offers many useful techniques and tools to bind compiled languages, such as ctypes, CFFI, f2py, Cython, Swig, and Boost.Python [7, 11-15]. In this section, we briefly introduce ctypes, f2py, and Boost.Python, which we utilized in our implementation.

The ctypes library is a standard Python package that enables one to directly load $\mathrm{C}$ and Fortran binary shared libraries and call their functions from within a Python program [7]. One advantage of using ctypes is that the source code of the libraries is unnecessary, so there is no need to prepare or modify source code. In contrast, ctypes performs few checks when a foreign function is called. Thus, for example, if wrong parameter types are passed to the function, the calling Python program will crash. Another problem is that it is somewhat difficult to call Fortran/C++ programs, because ctypes does not directly support calling conventions other than $\mathrm{C}$.

The f2py program is a Fortran-to-Python interface generator that generates stub code enabling one to directly call Fortran functions from Python programs [11]. More specifically, f2py is able to take Fortran source code and then generate a Python module that wraps functions (and subroutines) defined in the Fortran programs. One advantage of $\mathrm{f} 2 \mathrm{py}$ is that it is able to infer the argument types of the functions from the input source code and automatically generate the Python module. In addition, unlike ctypes, f2py inserts appropriate code to check the correctness of the parameter types that are passed to the wrapped functions. However, f2py cannot handle $\mathrm{C} / \mathrm{C}++$ programs (without modification), as it supports only Fortran. The interfaces of the Python modules generated by f2py are sometimes inefficient, incorrect, or both. This problem can be solved by explicitly defining appropriate interfaces with f2py's • pyf files.

Boost.Python is a wrapper library that encapsulates $\mathrm{C}++$ programs into Python modules that can be used from Python programs [14]. When provided with a configuration file describing the $\mathrm{C}++$ data structures to be exposed to Python, Boost.Python can generate appropriate source code (in the style of template programming) to wrap the data structures. Figure 4 shows an example, where the Boost.Python configuration code (bottom) describes the $\mathrm{C}++$ class (top). For example, the member variable atom type in the $\mathrm{C}++$ class Molecule can be accessed from Python, keeping the same data structure. That is, its corresponding Python class Molecule has the same member variable atom_type. The directives class_ and .def_readwrite indicate the class 
and member variable of the $\mathrm{C}++$ data structure, respectively, to be exposed to Python. The . def directive can be used for the member function of $\mathrm{C}++$. One advantage of Boost.Python is that, unlike ctypes or $\mathrm{f} 2 \mathrm{py}$, it is able to directly handle $\mathrm{C}++$ programs. However, conversely to f2py, Fortran programs are difficult to handle because the library is specialized for $\mathrm{C}++(\mathrm{C}$ programs can be handled by exporting them to $\mathrm{C}++$ ).

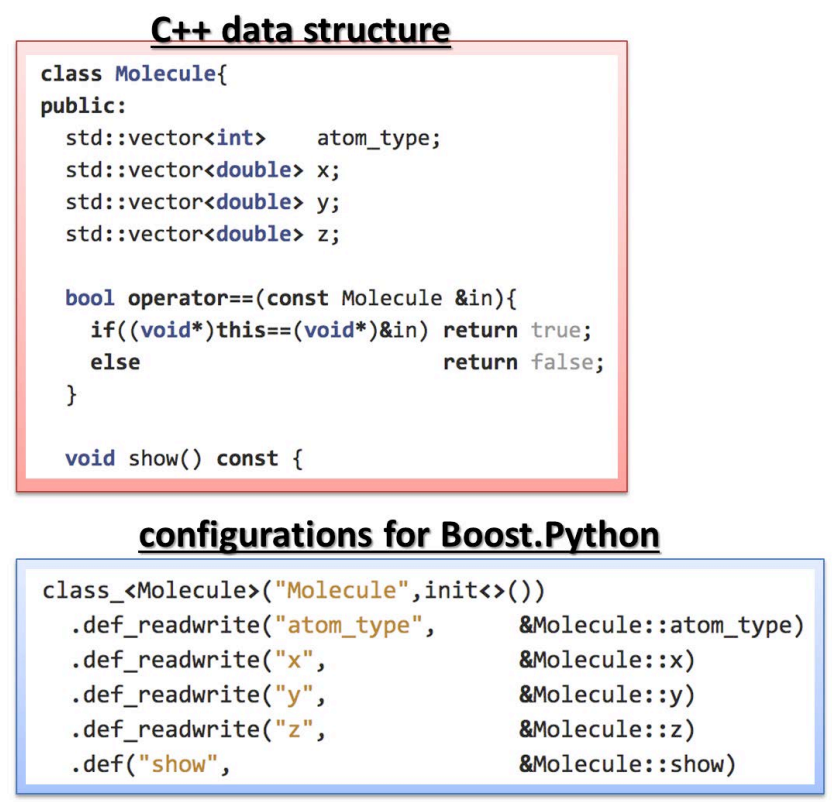

Figure 4. Boost.Python example.

\subsection{Our Implementation: Lotus}

Using the binding techniques described above, we have been developing Lotus, a Python-based quantum chemistry program. Figure 5 shows its overall structure.

As seen can be seen, the SCF method (described in Section 2 and outlined in Figure 2) is written in Python. In addition, the input/output processing is also written in Python. We have adopted JSON as an input file format and implemented its parser using the Python modules json and jsonschema. This eliminates the burden of implementing input parsers by hand.

On the other hand, the overlap, kinetic, nuclear attraction, Hartree, and exchange matrices, which are typical quantum chemistry calculations [8] and used in the SCF loop, are written in $\mathrm{C}++$ because of the heavy numerical burden that they present. The eigenvector calculations and matrix operations such as addition and multiplication are also implemented in Fortran and $\mathrm{C}++$, respectively. As discussed in subsections 2.1 and 2.2, however, these algorithms are exposed to Python by means of the binding techniques, and thus the SCF loop can utilize the exported routines. Moreover, we are able to replace the routines depending on the specific purpose by slightly modifying the SCF loop. In particular, the SCF loop is implemented as a function that takes functions as its arguments, so for example, the aforementioned matrix calculations are specified as input parameters to the SCF loop function, in the style of functional programming languages. Thus, the functions can be replaced simply by passing different functions to the SCF loop function. More detailed examples of this are described in subsection 4.2.

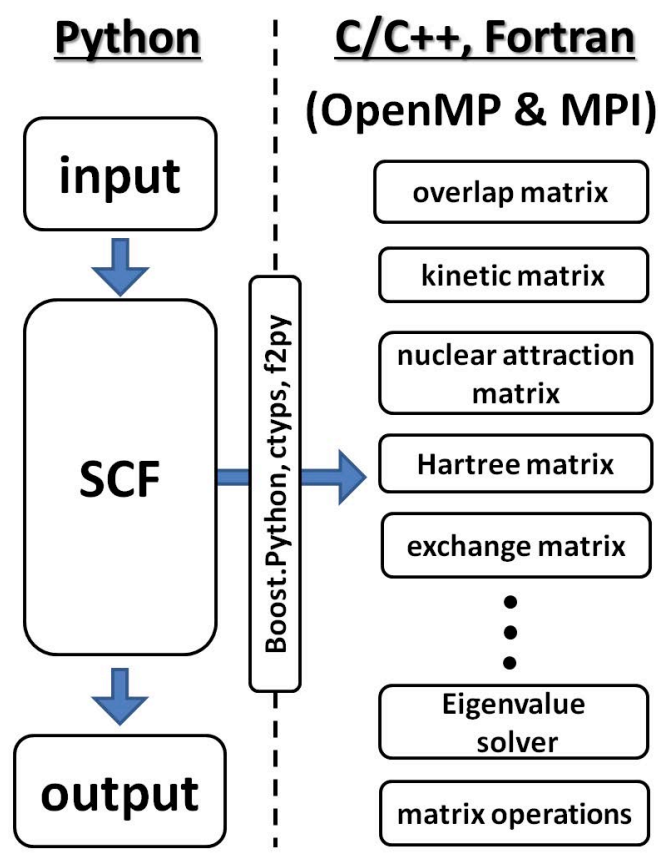

Figure 5. Overview of Lotus.

\subsubsection{Implementation notes for an HPC environment}

Here we describe some details of the implementation of Lotus for our HPC environments, the Fujitsu FX10 and the K computer [16] (the FX10 is a commercial model of the K computer). Although they are quite specific to our environment, we believe that other HPC environments may face similar problems, as such systems tend to have strong system dependencies, for example, systemdependent compilers, libraries, processor architectures, and so on.

One of the major problems we faced is the need to combine the use of two different compilers. Our FX10/K system requires Fujitsu's compiler (fcc) to achieve maximum performance for OpenMP and MPI. However, the Python runtime could not be built with Fujitsu's compiler. Therefore, we had to use the GNU C compiler (gcc) for the build. The problem is that the two compilers have their own approached to using OpenMP and MPI, and they are not aware of each other's activity. For example, in order to use OpenMP with fcc, we have to initialize OpenMP with the fcc initialization procedure, instead of gcc's (otherwise, fatal errors result). To avoid this problem, we modified the Python runtime (interpreter) so that it is able to initialize OpenMP and MPI appropriately. More specifically, we explicitly linked the static and shared libraries that are required to correctly initialize OpenMP and MPI for fcc.

Another problem is that some of the numerical libraries optimized and tuned for $\mathrm{FX} 10 / \mathrm{K}$ systems are provided as static builds. However, programs to be wrapped in Python modules have to be compiled as shared libraries. Thus, it is impossible to use the optimized/tuned routines from the shared libraries as is. To solve the problem, we linked the static libraries to the Python interpreter beforehand. One drawback of this approach is that the size of the interpreter increases by the sizes of the prelinked libraries, but it does enable the shared libraries to directly use the optimized/tuned routines.

Yet another problem is that the implementation of the standard template library (STL) of Fujitsu's C++ compiler (FCC) is not compatible with that of GNU $\mathrm{C}++$ compiler $(\mathrm{g}++)$. Therefore, 
Boost.Python, which depends on STL, needs to be built with FCC. However, the Boost library provides no official support for FCC. To solve the problem, we added several build configuration files for FCC to the Boost library.

\section{Preliminary Evaluation}

In this section, we present preliminary evaluations of the performance and the productivity aspects of Lotus.

\subsection{Performance}

We first assess the general performance degradation incurred by using Python to implement the SCF loop. Then, we evaluate the performance in our HPC environment (FX10).

\subsubsection{Performance Degradation}

To observe the effect on performance of writing the SCF loop in Python, we implemented the SCF method (Figure 2) in $\mathrm{C}++$ and compared its performance with that of the prototype implementation of Lotus. More specifically, we measured the execution times of both programs to calculate naphthalene molecule (Table 1) at the level of the Hartree-Fock (HF) method with the $6-31 \mathrm{G}^{*}$ basis set [8], using a workstation with two quadcore Xeon E5620 $2.4 \mathrm{GHz}$ CPUs. Both programs were parallelized with OpenMP.

The measured execution times are compared in the bottom row of Table 1. The prototype implementation of Lotus (at left) took $369.20 \mathrm{~s}$, while its $\mathrm{C}++$ implementation took $369.03 \mathrm{~s}$. These are the best results in 5 times execution (the variance is less than 1.0 s). This result indicates that implementing the SCF method in Python does not significantly degrade performance.

Table 1 also shows a breakdown of the execution times. The most time-consuming part in both programs was the calculation of the Fock matrix, which includes evaluation of the electron repulsion integrals (labeled "Fock"). Both programs spent about $99 \%$ of their execution time for these calculations. The second most timeconsuming part was the eigenvector calculations and direct inversion of the iterative subspace (DIIS) method [8] ("Eigen+DIIS"), which consumed about $0.8 \%$ of the execution time in both programs. The remaining calculations ("Others"), for example, computing one-electron operators, input/output processing, and so on, accounted for about $0.2 \%$ of the execution time in both cases. This also includes the overhead due to calling the $\mathrm{C}++$ functions from Python. As can be seen, there are no obvious differences in any part of the execution times. This is because the costs incurred by the binding techniques (described in subsection 3.1) amount to several milliseconds and the number of $\mathrm{C}++$ functions called from Python is small enough compared with the main body of the code.

Table 1. Execution times of quantum chemistry calculations for naphthalene

\begin{tabular}{ccc}
\hline \hline & with Python & without Python \\
\hline Fock [sec] & 365.58 & 365.65 \\
Eigen + DIIS [sec] & 2.97 & 2.84 \\
Others [sec] & 0.65 & 0.54 \\
\hline Total [sec] & 369.20 & 369.03 \\
\hline \hline
\end{tabular}

\subsubsection{Evaluation on an HPC Environment}

In this section, we describe the performance of the prototype implementation of Lotus on our HPC environment, described in subsection 3.2.1. Our FX10 consists of 96 nodes, each equipped with a Fujitsu SPARC64 IXfx CPU (1.848 GHz, 16 cores) and 32 GB main memory. Note that we used only 36 computing nodes in the following experiment.

Table 2 presents the execution times for calculations at the HF/6$31 \mathrm{G}^{*}$ level with the Lotus prototype. We employed three dry-ice models with different numbers of $\mathrm{CO}_{2}$ molecules. The times for calculating the Fock matrices were $7125,20,593$, and $68,494 \mathrm{~s}$ for models A, B, and C, respectively. As can be seen, this calculation was the most time-consuming part for all the three models, and the second greatest was the eigenvector calculations. These typical bottlenecks were implemented in $\mathrm{C} / \mathrm{C}++$ and Fortran, and the overhead incurred by the binding techniques are included in the "Others" entry, in the same way as in subsection 4.1.1. These results indicate that the overhead is small enough even in our FX10 HPC environment, given that it amounted to much less than $4.5 \%$.

Table 2. Execution times of quantum chemistry calculations for three dry-ice models

\begin{tabular}{cccc}
\hline \hline & Model A & Model B & Model C \\
\hline Num. of $\mathrm{CO}_{2}$ & 48 & 72 & 108 \\
Fock [sec] & 7125 & 20593 & 68494 \\
Eigen + DIIS [sec] & 1046 & 3029 & 9306 \\
Others [sec] & 365 & 1150 & 3686 \\
\hline Total Time [sec] & 8536 & 24772 & 81486 \\
\hline \hline
\end{tabular}

\subsection{Productivity}

We argue here that our approach can improve productivity in the development of quantum chemistry simulations by showing that our Python-based quantum chemistry program Lotus can be easily modified and extended by wrapping existing libraries as Python modules or using existing Python modules. As described in Section 1 , it is difficult to conduct practical quantum chemistry simulations with a single program package because many theories and complex algorithms are involved, depending on the various molecules and materials under consideration. Thus, ease of integrating multiple program packages is important to improving productivity in the context of such simulations. One of us (T. S.) has conducted practical scientific studies with Lotus [17-21]. Based on our experience, we believe that the Python-based approach described in this paper is not only practical from the viewpoint of performance, but also effective from the standpoint of productivity.

More specifically, we examine three case studies that involve modifying and extending Lotus to integrate it with three different projects: SMASH [2], EigenExa [22], and ASE [23]. Figure 6 provides a structural overview of the integration: the main routine (the SCF algorithm) is written in Python, and the intensive numerical computations (including external libraries) are wrapped as Python modules using the binding techniques described above. 


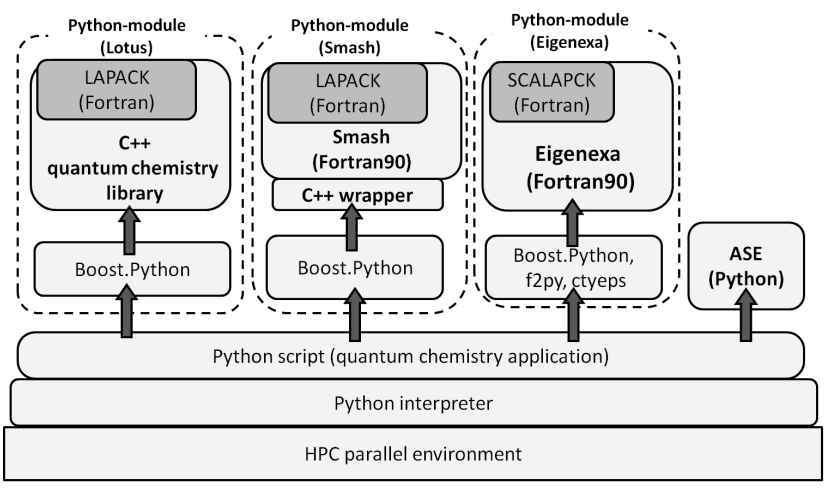

Figure 6. Overview of integrating Lotus with external libraries.

\subsubsection{Integration with SMASH}

SMASH [2] is a quantum chemistry program written in Fortran90. SMASH is heavily tuned for massively parallel supercomputers such as the K computer [16]. It should be noted that Lotus and SMASH have been developed entirely independently. Thus, these two quantum chemistry programs have different characteristics and computational methodologies. For example, Lotus is able to deal with materials under periodic boundary conditions, which are not supported by SMASH.

Nevertheless, we were able to successfully integrate Lotus with SMASH to execute quantum chemistry calculations, simply by importing its Python module into the main script and replacing the original functions with those from SMASH. Based on our experience, we believe that the integration was much easier and more flexible than a direct approach at the level of a compiled language.

In order to create a Python module of SMASH, we wrote a small wrapper (less than 160 lines) binding Fortran90 to $\mathrm{C}++$, because Boost-Python cannot directly handle Fortran90. Moreover, we had to refactor SMASH (about 3200 lines of code modification) because several functions cannot be directly exported due to their strong mutual dependence.

\subsubsection{Integration with EigenExa}

In the SCF algorithm, the calculation of eigenvalues and eigenvectors is a very time consuming step [8] (as shown in subsection 4.1), and thus it is important to optimize or tune the calculations to improve the performance of quantum chemistry simulations. However, both our (original) Lotus and SMASH employ the LAPACK library for the eigenvector calculations [24], which supports only single-core CPUs.

To make full use of parallel supercomputers, it is necessary to employ efficient parallel eigenvalue solvers. For this purpose, we utilized EigenExa [22], a high-performance solver written in Fortran90 that is targeted at massively parallel supercomputers, particularly the K computer. To integrate EigenExa into Lotus, we first modified EigenExa so that we could build it as a shared library, because the original EigenExa was available only as a static library. Then we wrapped the shared library in a Python module. We examined three binding techniques: ctypes, f2py, and Boost.Python (explained in subsection 3.1). In the Boost.Python case, we prepared $\mathrm{C}++$ wrappers (less than 200 lines) for the Fortran90 source code of EigenExa (in the same way as for SMASH). By contrast, ctypes and f2py can deal directly with Fortran programs, though ctypes and f2py required less than 180 and 140 lines of data conversion code, respectively. We confirmed that these three binding techniques are able to construct a Python module for EigenExa. Integration of Lotus and EigenExa was achieved by simply importing the Python module into the main script and replacing the related calculation routines with those from EigenExa.

\subsubsection{Integration with ASE}

ASE (Atomic Simulation Environment) [23] is a set of Python modules that provides common tools for atomic-scale simulations, such as global and local optimizers, transition-state search based on the nudged elastic band method, and integrators for molecular dynamics. Unlike the examples described above, ASE is written in pure Python. The computational costs of quantum chemistry methods are different substantially among algorithms. For example, Lotus and SMASH mainly target the electronic structure calculations, which incur huge computational costs. Conversely, ASE provides computationally lightweight algorithms that execute quickly enough even if written in pure Python. The integration of Lotus and ASE was achieved by simply importing the Python modules of ASE in the main Lotus script. In addition, we were successfully able to use the optimizers provided by ASE for the Lotus SCF algorithm (31 lines of Python code).

\section{Concluding Remarks}

We have presented a hybrid approach to improving productivity in quantum chemistry program development, which combines a dynamic scripting language with compiled languages and was used to develop a quantum chemistry program and illustrate the advantages over traditional development.

In our approach, computationally expensive algorithms are implemented in compiled languages such as $\mathrm{C} / \mathrm{C}++$ and Fortran and bound to Python modules, which can be called from ordinary Python scripts. For example, we implemented the overlap, kinetic, nuclear attraction, Hartree, and exchange matrices in $\mathrm{C}++$. We also employed compiled languages for eigenvector calculations and matrix operations.

However, the main routines of the SCF algorithm can be written entirely as a simple Python script without sacrificing performance, as long as it conforms to the program structure shown in Figure 5. In other words, this approach, in which quantum chemistry matrix calculations are separated into external modules written in compiled languages, represents a promising strategy to implement quantum chemistry algorithms in a hybrid manner with respect to computational performance.

Obviously, dynamic programming languages can improve productivity in the context of program development. They enable one to rapidly develop complex simulation programs without time-consuming compilation. Moreover, the behavior of a simulation program can even be modified at run time by simply editing scripts. Such a dynamic approach to simulation programs has not been well studied in quantum chemistry, because only compiled languages are supported in many case. The hybrid approach might help to develop novel theories in the future.

Last but not least, our approach is not limited to the combination of Python and compiled languages such as $\mathrm{C} / \mathrm{C}++$ and Fortran. Any combination is possible so long as language binding mechanisms are available. 


\section{References}

[1] Valiev, M., Bylaska, E. J., Govind, N., Kowalski, K., Straatsma, T. P., Van Dam, H. J. J., Wang, D., Nieplocha, J., Apra, E., Windus, T. L. and de Jong, W. NWChem: A comprehensive and scalable open-source solution for large scale molecular simulations. Comput Phys Commun, 181 (May 13 2010), 1477.

[2] Ishimura, K. SMASH, http://smash-qc.sourceforge.net.

[3] Hanenberg, S. An experiment about static and dynamic type systems: doubts about the positive impact of static type systems on development time. In Proc. of the ACM international conference on Object oriented programming systems languages and applications (OOPSLA 2010). pp. 2235. http://doi.acm.org/10.1145/1869459.1869462

[4] Delorey, D. P., Knutson, C. D., and Chun, S. Do Programming Languages Affect Productivity? A Case Study Using Data from Open Source Projects. First International Workshop on Emerging Trends in FLOSS Research and Development (FLOSS'07: ICSE Workshops 2007), pp. 8.

[5] Muller, R. P. PyQuante, http://pyquante.sourceforge.net.

[6] Plimpton, S. Fast Parallel Algorithms for Short-Range Molecular-Dynamics. J Comput Phys, 117 (Mar 1 1995), 1.

[7] Python, https://www.python.org.

[8] Jensen, F. Introduction to Computational Quantum Chemistry. John Wiley \& Sons Ltd, Chichester, 1999.

[9] Shimazaki, T. and Kubo, M. Efficient density functional theory calculations with weak hydrogen quantum effect: Electron density analysis. Chem Phys Lett, 525-26 (Feb 16 2012), 134

[10] Shimazaki, T. and Asai, Y. Electronic Structure Calculations under Periodic Boundary Conditions Based on the Gaussian and Fourier Transform (GFT) Method. J Chem Theory Comput, 5 (Nov 25 2009), 136.

[11] CFFI, https://cffi.readthedocs.org/en/latest/

[12] NumPy, http://www.numpy.org.

[13] Cython, http://docs.cython.org.
[14] Swig, http://www.swig.org.

[15] Abrahams, D. Boost.Python, http://www.boost.org.

[16] Miyazaki, H., Kusano, Y., Okano, H., Nakada, K., Seki, K., Shimizu, T., Shinjo, N., Shoji, F., Uno, A., and Kurokawa, M. K computer: 8.162 PetaFLOPS massively parallel scalar supercomputer built with over 548k cores. In Proc. of the 2012 IEEE International Solid-State Circuits Conference (ISSCC) Digest of Technical Papers, pp. 192-194, 2012.

[17] Shimazaki, T., Kosugi, T. and Nakajima, T. RangeSeparation Density-Fitting Band Structure Calculation with Gaussian Auxiliary Function. J Phys Soc Jpn, 83 (Apr 4 2014), 054702.

[18] Shimazaki, T. and Nakajima, T. Dielectric-dependent screened Hartree-Fock exchange potential and Slater-formula with Coulomb-hole interaction for energy band structure calculations. J Chem Phys, 141 (Sep 21 2014), 114109.

[19] Shimazaki, T. and Nakajima, T. Theoretical study of a screened Hartree-Fock exchange potential using positiondependent atomic dielectric constants. J Chem Phys, 142 (Feb 21 2015), 074109.

[20] Shimazaki, T. and Nakajima, T. Theoretical study of exciton dissociation through hot states at donor-acceptor interface in organic photocell. Phys Chem Chem Phys, 17 (Apr 16 2015), 12538 .

[21] Shimazaki, T. and Nakajima, T. Gaussian-based cutoff scheme on Hartree-Fock exchange term of dielectricdependent potential. Chem. Phys. Lett., 634 (June 9 2015), 83.

[22] EigenExa: a High performance eigen-solver http://www.aics.riken.jp/labs/lpnctrt/EigenExa_e.html.

[23] Bahn, S. R. and Jacobsen, K. W. An object-oriented scripting interface to a legacy electronic structure code. Comput Sci Eng, 4 (Oct 7 2002), 56.

[24] Anderson, E., Bai, Z., Bischof, C., Blackford, S., Demmel, J., Dongarra, J., Du Croz, J., Greenbaum, A., Hammarling, S., McKenney, A. and Sorensen, D. LAPACK User's Guide. Society for Industrial and Applied Mathematics, 1999. 